Supplementary Material for the article:

\title{
Universality in spatio-temporal high-mobility domains across the glass transition from bulk polymers to single chains
}

\author{
Lorena Alzate-Vargas ${ }^{1}$, Nicolas Onofrio ${ }^{2}$, and Alejandro Strachan ${ }^{1}$ \\ ${ }^{1}$ School of Materials Engineering and Birck Nanotechnology Center, \\ Purdue University, West Lafayette, Indiana 47907 USA. \\ ${ }^{2}$ Department of Applied Physics, The Hong Kong Polytechnic University, Hong Kong, SAR
}

The calculated glass transition values for the studied bulk polymers using two fitting procedures are shown in Table S1, we also report the experimental values accounting for cooling rate effects using the widely used values of $3 \mathrm{~K}$ and $5 \mathrm{~K}$ per order of magnitude change in cooling rate. The discrepancies in the averaged glass transition temperature as predicted by two fits with respect to the $3 \mathrm{~K}$ per magnitude value are $49 \mathrm{~K}$ overestimation in PMMA ( $\sim 12 \%$ of the experimental value), $37 \mathrm{~K}$ overestimation in PS ( $\sim 9 \%$ of the experimental value), $32 \mathrm{~K}$ underestimation in $\mathrm{PP}(\sim 11 \%$ of the experimental value), and $31 \mathrm{~K}$ underestimation in PE ( $\sim 13 \% \%$ of the experimental value).

Table S1. Comparison of experimental and predicted glass transition temperatures for bulk polymers using two fitting procedures: bilinear and hyperbola.

\begin{tabular}{ll|c|c|c|c}
\multicolumn{2}{c|}{$T_{g}[\mathrm{~K}]$} & PMMA & PS & PP & PE \\
\hline \multirow{2}{*}{ Predicted } & Bilinear & 453 & 442 & 246 & 195 \\
\cline { 2 - 6 } & Hyperbola & 479 & 456 & 275 & 208 \\
\cline { 2 - 6 } & Average & 466 & 449 & 261 & 202 \\
\hline Experiment & 378 & 373 & 253 & 193 \\
\hline Experiment corrected 3 K & 417 & 412 & 292 & 232 \\
\hline \multicolumn{2}{l|}{ Experiment corrected 5 K } & 443 & 438 & 318 & 258
\end{tabular}

The RDFs between torsion centers were computed for PMMA and PS at $450 \mathrm{~K}$ and for PP and PE at 260 $\mathrm{K}$ and $200 \mathrm{~K}$ respectively as shown in Fig. S1. The sharp peaks at short distances correspond to nearby torsions along the polymer backbone, the broader peaks correspond to non-bond distances. As expected, observation of percolation requires cutoffs long enough to capture torsions in nearby chains. 

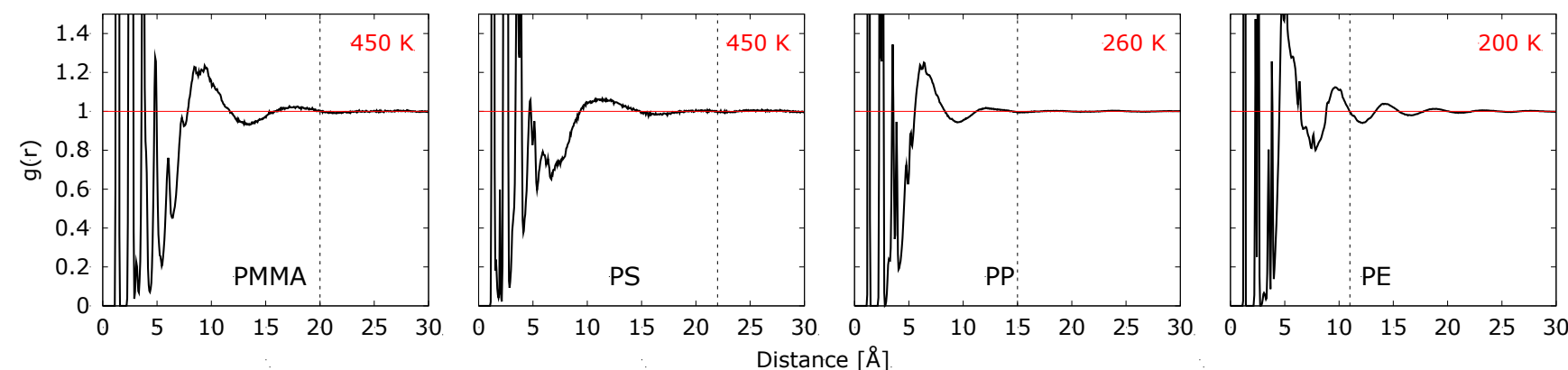

Figure S1. Radial distribution function of backbone torsions at $450 \mathrm{~K}$ in bulk PMMA and PS, $260 \mathrm{~K}$ in PP and $200 \mathrm{~K}$ in PE.
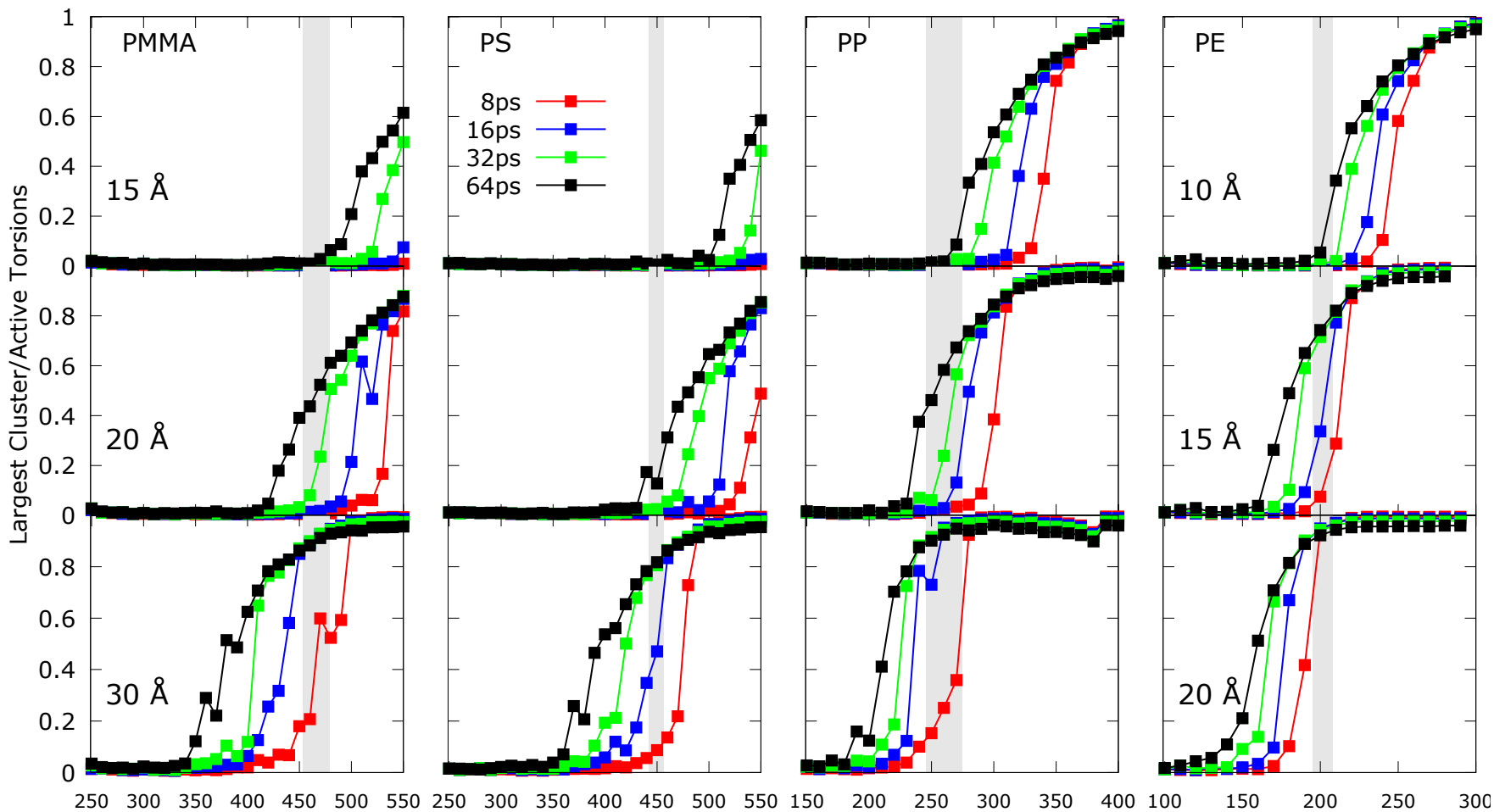

Temperature $[\mathrm{K}]$

Figure S2. Largest cluster normalized by active torsions as a function of temperature with different time cutoffs and spatial cutoffs for bulk samples consisting of 40 chains each 100 monomers. Columns refer to polymers and rows to cutoffs (same cutoffs are used for PMMA, PS and PP).

Local dynamics of polymers can be probed by monitoring the torsional autocorrelation function (TACF) defined as 


$$
\operatorname{TACF}(t)=\frac{\langle\cos \theta(t) \cos \theta(0)\rangle-\langle\cos \theta(0)\rangle^{2}}{\left\langle\cos ^{2} \theta(0)\right\rangle-\langle\cos \theta(0)\rangle^{2}}
$$

where $\theta(t)$ and $\theta(0)$ are the backbone dihedral angles at time $t$ and 0 respectively. The time decay of the TACF, associated with the segmental relaxation can be fitted to a Kohlrausch-Williams-Watts (KWW) function

$$
f(t)=A \exp \left[-\left(\frac{t}{\tau}\right)^{\beta}\right]
$$

here $\tau$ is the characteristic time associated with the $\alpha$-relaxation. The calculated $\alpha$-relaxation correlation times for bulk polymers, as a function of temperature, are shown in Fig. S3.

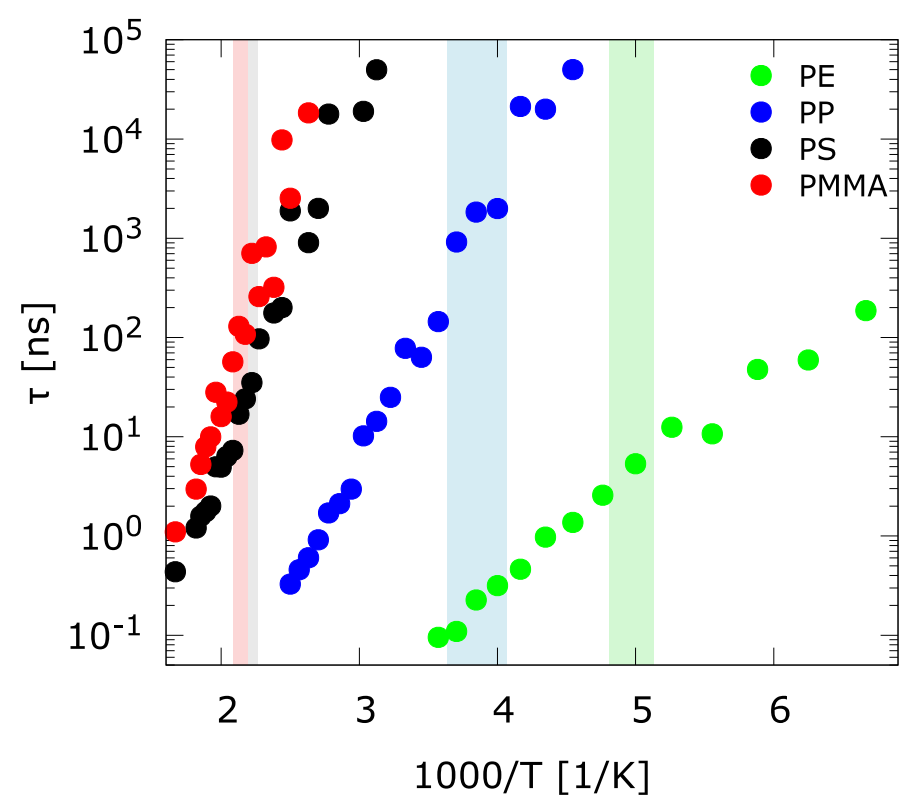

Figure S3. Characteristic $\alpha$-relaxation (correlation) time $(\tau)$ for bulk samples calculated from fitting the torsional autocorrelation function to a Kohlrausch-Williams-Watts (KWW) function as a with respect of the inverse temperature. Colored regions indicate the glass transition regime.

We find it instructive to visualize correlations between active torsions using $t_{c}=32$ and 64 ps displayed in Fig. S4 for bulk polymers and Fig. S5 for PMMA samples. We show all active torsions as a function of time, points mark an active torsion and the torsion index (used in the vertical axes) is unique and assigned sequentially as torsions become active. Active torsions are colored according to the cluster they belong to. We find significant correlations between active torsions in the glassy state, denoted by groups of dots with the same color. 


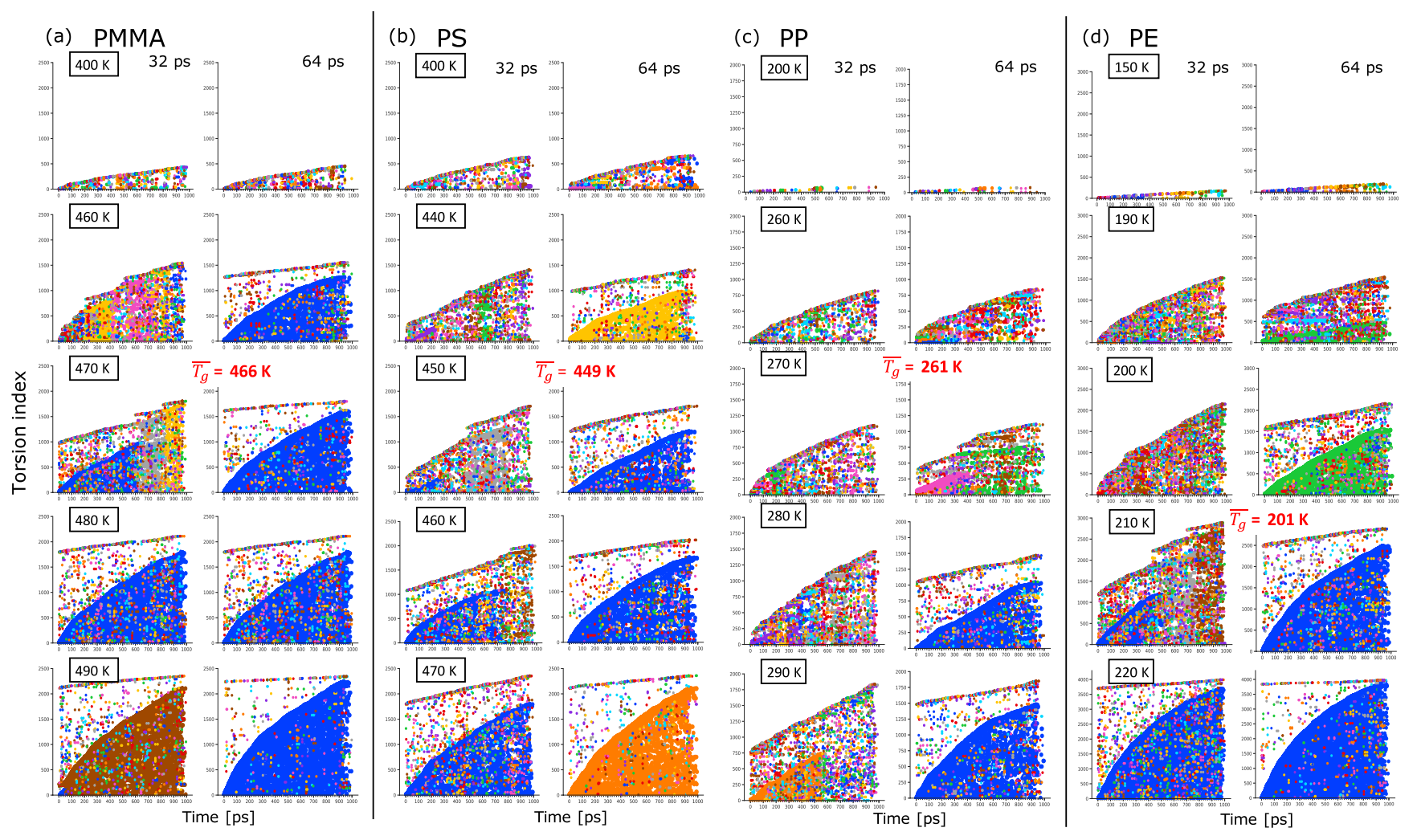

Figure S4. Cluster evolution in a range temperature in the vicinity of $T_{g}$, active torsions are index in order of appearance for (a) PMMA, (b) PS, (c) PP and (d) PE.

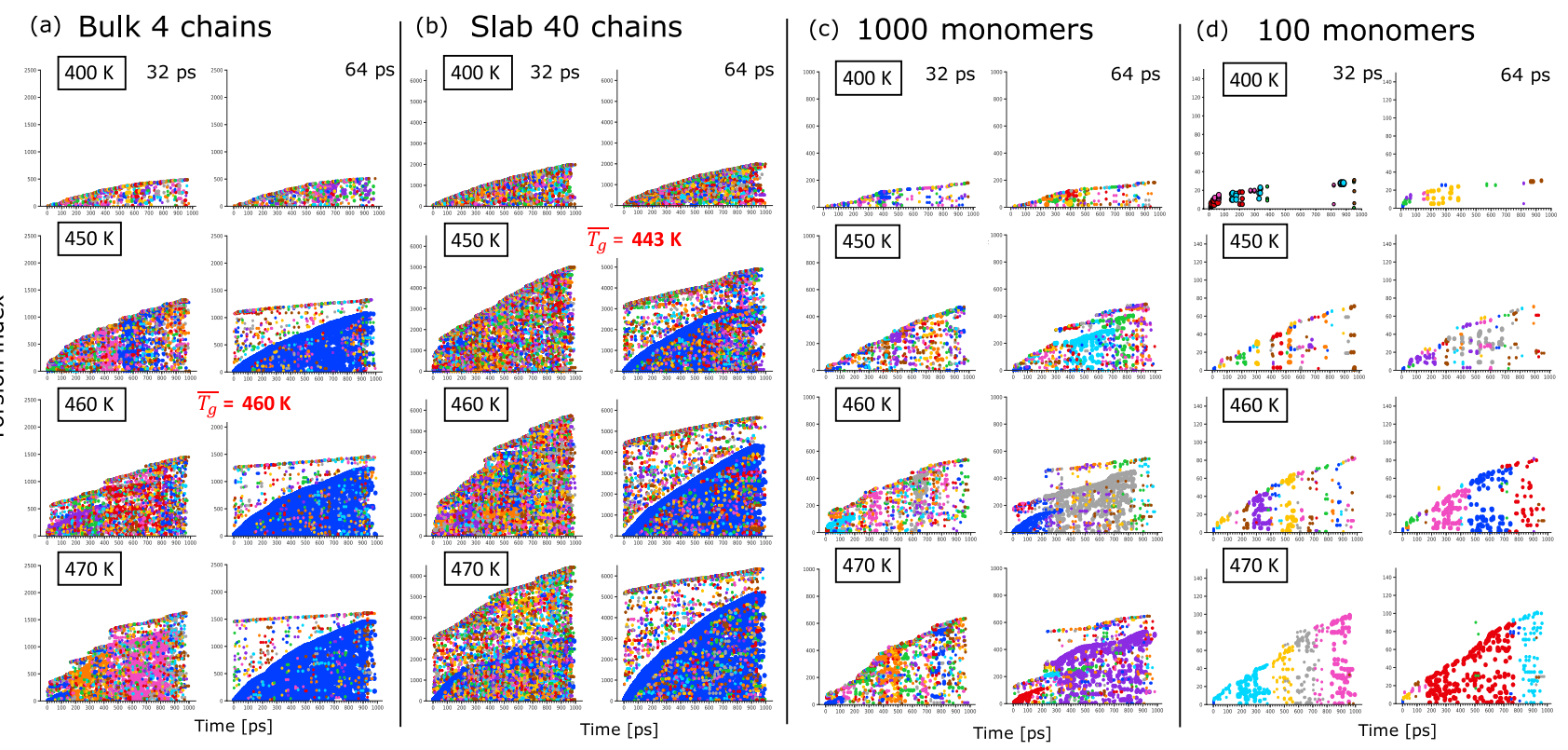

Figure S5. Cluster evolution in a range temperature in the vicinity of $T_{g}$ for PMMA samples. 